\title{
Individual differences in secondary task performance
}

\author{
MARCY LANSMAN and EARL HUN'T \\ University of Washington, Seattle, Washington 98195
}

\begin{abstract}
The purpose of this research was to use secondary task performance as a predictor of performance on a difficult primary task. Reaction time (RT) to secondary probes that occurred during the rehearsal period of an easy memory task were used to measure spare capacity associated with the memory task. This measure was then correlated with performance on a harder version of the same memory task. Experiments 1-4 involved a paired associate memory task. Probe RT was sensitive to the difficulty of the paired associate task, and analysis of individual differences showed that probe RT during the easy version of the task was correlated with performance on a harder version of the task. Experiment 4 also utilized a spatial memory task. Probe RT was less sensitive to the demands of the spatial memory task, and in that case, the "easy-to-hard" prediction was not successful.
\end{abstract}

This article is concerned with performance on a dual task in which one component is designated as primary and the other as secondary. Suppose that there is something analogous to "mental energy," an attentional resource that is required for a variety of tasks (Kahneman, 1973). If two tasks are performed concurrently, with one designated as primary and the other as secondary, then the resource demands of the primary task should be fulfilled first. Therefore, secondary task performance should provide a measure of the spare capacity not required by the primary task (Kerr, 1973). Secondary task performance should decrease as the capacity demands of the primary task increase. The most common uses of secondary task measures have been to compare the demands of various stages in the execution of a primary task (e.g., Johnston, Greenberg, Fisher, \& Martin, 1970; Logan, 1978; Posner \& Boies, 1971) and to compare the demands of the same primary task under various conditions (e.g., Britton, Westbrook, \& Holdredge, 1978; Martin, 1970). Another use of secondary task measures has been to differentiate among individuals who vary in skill on the primary task. Those individuals who are highly skilled on the primary task should require a smaller proportion of their total resources to perform the task and should, therefore, have more spare capacity available to perform the secondary task. This use of secondary task measures has

This research was supported by the Office of Naval Research through Contract N00014-77-C-0225 to the University of Washington, Earl Hunt, principal investigator. We would like to thank Beth Kerr for advice on design and interpretation and comments on an earlier draft. We would also like to thank Janet Davidson, Simon Farr, and Colene McKee for assistance in executing and analyzing these experiments. Requests for reprints should be sent to Marcy Lansman, Department of Psychology, NI-25, University of Washington, Seattle, Washington 98195. been reported more often in the applied than in the experimental literature (e.g., Brown, 1968).

The assertion that primary and secondary tasks compete for mental resources has a further implication for individual differences in secondary task performance. Secondary task measures associated with an easy version of the primary task should predict performance on a harder version of the same primary task. Consider two people, one skilled at primary task performance and the other less skilled. Both individuals may perform an easy version of the primary task at relatively high levels of accuracy. Presumably, though, the less skilled individual is using more mental resources to achieve this level of accuracy. Thus the two persons will perform at different levels on a secondary task. If this is the case, secondary task performance when the primary task is easy should predict primary task performance at a higher level of difficulty. We shall refer to this prediction as the "easy-tohard prediction." Such a measurement technique might be useful in job training, since it would allow one to predict, on the basis of performance in a routine job situation, how well a trainee might handle the heavier demands of an emergency. The purpose of the research reported here was to test the validity of the easy-to-hard prediction for several dual task combinations in which the primary component was a memory task and the secondary component was a probe reaction time (RT) task. If the technique can be shown to be effective for these laboratory tasks, it would be reasonable to apply it in more realistic situations.

Easy-to-hard prediction is based on a simple model of dual task performance. According to this model, each subject has a fixed supply of mental resources. ${ }^{1}$ Primary and secondary tasks compete for these resources, and performance on each task improves as more resources are devoted to it. During the dual task involving the easy 
version of the primary task, subjects allot enough resources to the primary task to perform that task adequately, and they devote their remaining resources to the secondary task. Thus, secondary task performance can be used as a measure of spare capacity (i.e., capacity not required by the easy version of the primary task). Individual differences in secondary task performance should reflect individual differences in spare capacity and, hence, should identify people who will do well or poorly when the primary task becomes harder. The assumptions underlying this prediction are that the hard version of the primary task requires more resources than the easy version and that an individual's performance on the hard version of the primary task is limited by total available resources. Those individuals who have more spare capacity during the easy version of the primary task should have more resources available to meet the increased resource demands of the primary task and should thus perform the task at a higher level. Hence, performance on the secondary task during the easy version of the primary task should be positively correlated with performance on the hard version of the same primary task.

Our discussion so far has ignored several complicating issues. First, individual differences in spare capacity as measured by the secondary task may reflect individual differences in either total capacity or capacity demands of processes involved in the primary task. To use the electrical analogy, the "spare capacity" of a household electrical circuit is a function of both the capacity of the incoming line and the power requirements of the appliances being used. Similarly, capacity available to the secondary task may be a function of both the total capacity of the system and the efficiency (in terms of mental capacity) with which a person carries out the primary task. Our experiments do not allow us to distinguish between these two sources of variation. A further issue is that secondary task performance may reflect other factors besides spare capacity. These "structural" factors should affect single task as well as dual task performance. In an attempt to remove variation attributable to structural factors, we partialled out single task performance in computing the easy-to-hard correlation. Finally, people may differ in their allocation strategies: Some may devote a greater proportion of their capacity to the secondary task than others do. Although we could not eliminate this possibility entirely, the data suggest that differences in allocation strategies are not an important determinant of correlational patterns.

The easy-to-hard prediction is based on the assumption that the hard version of the primary task requires more general capacity than the easy version. Whether this assumption is met depends on how the primary task is made more difficult. Theoretically, the difficulty of a task can be increased in several ways. The harder version may involve the same mental processes as the easy version, but it may require that they be carried out faster or more often. We chose easy and hard tasks that seemed to fall in this category. Note that even in this case, the two versions of the task may not be highly correlated, since capacity limitation may determine performance on the hard but not on the easy version. Alternatively, the hard version of a task may involve processes not required by the easy version, and these additional processes may increase the capacity demands of the task. In both these cases, the easy-to-hard prediction should be valid. Finally, however, a task can be made more difficult by challenging the limits of a specific system rather than increasing the general capacity demands. For example, a visual task could be made more difficult by shortening the duration of a display so that subjects must retrieve information from a fading icon. In this case, we would not expect general capacity limitations to determine performance. To use Norman and Bobrow's (1975) terminology, the task would be data limited rather than resource limited. Here, the easy-to-hard technique would be inappropriate. The experiments reported in this paper may be looked upon as a demonstration that it is possible to complicate a primary task in a reasonable way such that the easy-tohard prediction is valid. It cannot be argued that the technique will work for all pairs of easy and hard tasks.

In Experiments 1-3, the primary task was a continuous paired associate learning task similar to that used by Atkinson and Shiffrin (1968). In Experiment 4, we also used a spatial task that involved holding in memory a random pattern of plus signs. In all cases, the secondary task required the subject to make a simple response to a visual or auditory stimulus. The stimulus for the secondary task could occur only during the rehearsal period of the primary task. Thus, the subject was never required to respond to the two tasks during the same interval, and response interference was avoided.

The pattern of interference manifested in secondary probe RT may depend on the exact type of secondary probe task employed (McLeod, 1977, 1980; Schwartz, 1976). Consequently, we combined the verbal paired associate memory task with two different probe tasks. The first, which required a manual response to a visual probe, was selected to involve specific resources as distinct as possible from those involved in rehearsal of verbal material. The second, which required a vocal response to an auditory probe, was selected to involve the same auditory-vocal system utilized by verbal rehearsal (Baddeley, 1976). If interference between the verbal memory task and the probe task involves competition for a specific system, then only the auditory-vocal task should be sensitive to the demands of the verbal memory task. But if interference reflects demands on a more general resource pool, then both probe tasks should vary with the demands of the verbal memory task.

The first experiment, which did not involve analysis of individual differences, was intended to establish that the paired associate task and the two probe tasks did indeed compete for resources. In the second and third experiments, the validity of easy-to-hard prediction was 
tested using paired associate learning as the primary task. Experiment 2 involved the visual-manual probe task, and Experiment 3 involved the auditory-probe task. In Experiment 4 , the easy-to-hard prediction technique was extended to the spatial memory task.

\section{EXPERIMENT 1}

\section{Method}

Subjects. Subjects were 20 male and 16 female freshmen at the University of Washington. Each subject participated for four $1 \mathrm{~h}$ sessions and was paid $\$ 3 / \mathrm{h}$.

Apparatus. Presentation of stimuli and recording of responses were under the control of a Data General Corporation NOVA 3 computer. Visual stimuli were presented on Tektronix 604 oscilloscopes. Tones were generated by a Wavetek waveform generator (Model 159-002) and presented through Telephonics TDH49P headphones. One to six subjects were run simultaneously in separate soundproofed booths. Each was seated in front of a $10 \times 13 \mathrm{~cm}$ oscilloscope screen with fingers resting on eight pushbutton-style response keys. The rate of progress of each subject through the task was independent of the progress of other subjects.

Tasks. Primary task. The primary task was a continuous paired associate recall task in which subjects kept track of two, three, four, five, or seven letter-digit pairs. A typical sequence of events for the two-pair condition is illustrated in Table 1. The subject initiated the block by pressing a key. Then, each of the letters appeared for $3 \mathrm{sec}$, paired with a randomly chosen digit (e.g., "A = 3"). After this initial presentation of all letter-digit pairs, each trial consisted of a question involving one of the letters (e.g., "A = ?"), followed by a new pair involving that same letter (e.g., "A =4"). On each trial, the letter to be queried was chosen randomly from the entire set.

The correct response to a question was the number with which the letter had last been paired. Subjects responded by pressing one of eight numbered keys. After the response, a 1-sec feedback message ("Right" or "Wrong") appeared on the screen. If a subject failed to respond for $10 \mathrm{sec}$, the message "Too Slow" appeared on the screen and an error was recorded. Following the feedback message, a new pair was presented. The letter just queried was paired with a new digit, which was randomly chosen from the digits $1-8$, with the restriction that it could not be the same as the digit last paired with that letter. The new pair remained on the screen for a 3 -sec rehearsal interval and was followed by the question for the next trial. Each subject had a different sequence of letter-digit pairs.

At the end of each block, subjects received feedback concerning the percent of digits they had recalled correctly.

Secondary task. In the secondary task, the subject responded as quickly as possible to a probe stimulus presented during the rehearsal interval. The visual probe consisted of four asterisks, which appeared immediately above a letter-number pair. The auditory probe was a tone presented to the subject through headphones. Subjects responded to visual probes by pressing any of the eight keys. They responded to auditory probes by saying the syllable "bop" into a microphone.

Probes were always presented during the rehearsal interval for a new pair (never during a question), so that the subject was never required to respond to two tasks during the same interval. No probes occurred during the initial presentation of pairs in a block. An equal number of probes occurred at 500, 1,000, and $1,500 \mathrm{msec}$ following the onset of a new letter-digit pair. Probes were presented on three-fourths of the trials in a block. The order of probe and no-probe trials was independently randomized for each subject, as was the order of the three probe intervals.

The probe was removed as soon as the subject responded or after $1,500 \mathrm{msec}$ if no response occurred. Subjects did not receive feedback concerning probe RTs.

Procedure. The experiment was conducted on 4 consecutive days, $1 \mathrm{~h} /$ day. During the $1 \mathrm{st}$ day, subjects were acquainted with all the conditions of the experiments, but no data were recorded. Each of the following 3 days was devoted to one of three conditions: primary task alone, primary task with visual probes, or primary task with auditory probes. The 36 subjects were divided into six groups of 6 subjects each, and each of these groups received one of the six possible orders of the three conditions.

Primary task alone. In this condition, subjects received the continuous paired associate task alone; no probes were presented. There were five blocks of 36 trials each. During each block, subjects kept track of two, three, four, five, or seven letter-digit pairs. The order of blocks was counterbalanced across the six groups.

Primary task with visual probes. In this condition, subjects performed both the paired associate and the visual probe tasks. There were six blocks of trials, one each for two, three, four, five, and seven pairs, and an additional secondary task control condition, during which subjects ignored the letter-digit pairs and responded only to the probes. During the control condition, questions and new pairs occurred just as in the other blocks, but the question was removed after 1 sec and the subject was not required to respond to it.

Primary task with auditory probes. In this condition, subjects performed both the paired associate and the auditory probe task. In all other respects, this condition was identical to the primary task with visual probes condition.

\section{Results}

Probe RT. For each subject, mean RT to the probe was computed for each condition. Mean RTs across subjects are shown in Figure 1. Each point in Figure 1 is

Table 1

Sequence of Events for the Two-Item Memory Load, Experiment 1

\begin{tabular}{|c|c|c|}
\hline Event & Display & Duration \\
\hline Sequential presentation of initial pairs. & $\begin{array}{l}A=7 \\
B=3\end{array}$ & $\begin{array}{l}3 \mathrm{sec} \\
3 \mathrm{sec}\end{array}$ \\
\hline Question. The correct answer is 3 . & $\mathrm{B}=$ ? & Subject paced \\
\hline $\begin{array}{l}\text { Rehearsal interval. Letter just queried } \\
\text { is paired with a new number. }\end{array}$ & $B=4$ & $3 \mathrm{sec}$ \\
\hline $\begin{array}{l}\text { Probe. A probe may occur } 500,1,000 \text {, or } \\
1,500 \text { msec after presentation of a new pair. }\end{array}$ & $\begin{array}{l}(* * * *) \\
\mathrm{B}=4\end{array}$ & $\begin{array}{l}\text { Probe is presented until subject responds, } \\
\text { for a maximum of } 1,500 \mathrm{msec} \text {. }\end{array}$ \\
\hline Question. The correct answer is 7. & $A=?$ & Question remains on screen until subject responds. \\
\hline $\begin{array}{l}\text { Rehearsal interval. Letter just queried } \\
\text { is paired with a new number. }\end{array}$ & $A=5$ & $3 \mathrm{sec}$ \\
\hline
\end{tabular}




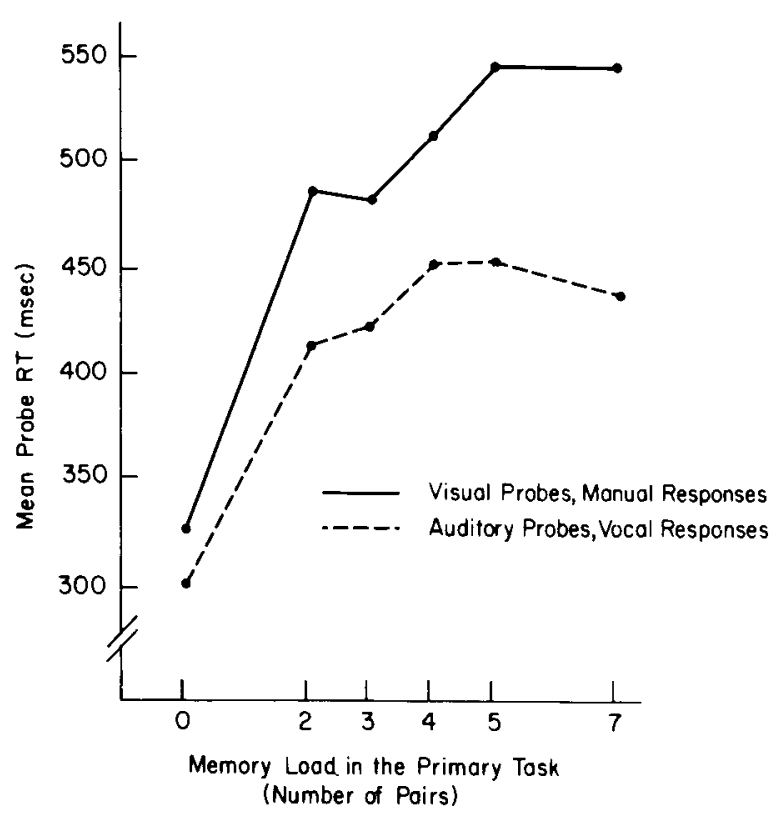

Figure 1. Mean probe RT for the visual-manual and auditoryvocal secondary tasks as a function of memory load in the primary task, Experiment 1 .

based on approximately 27 data points/subject. (Subjects failed to respond to $.5 \%$ of the auditory probes and $.8 \%$ of the visual probes.) The RT data were analyzed using a repeated-measures analysis of variance (ANOVA) in which the within-subjects factors were memory load (zero, two, three, four, five, or seven pairs) and probe task (manual response to a visual probe or vocal response to an auditory probe). The main effect of memory load was significant $[F(5,175)=71, \mathrm{MSe}=4,832, \mathrm{p}<.001]$, as were the main effect of probe task $[F(1,35)=22$, $\mathrm{MSe}=24,000, \mathrm{p}<.001]$ and the interaction $[\mathrm{F}(5,175)=$ $4.5, \mathrm{MSe}=3,315, \mathrm{p}<.001]$. Responses to the probe were longer when subjects were maintaining larger memory loads, and they were longer for the visualmanual probe task than for the auditory-vocal probe task. RTs to the visual-manual probe task increased more sharply with memory load than did RTs to the auditoryvocal probe task.

The difference between RT in the control condition and RT in the various memory load conditions was much greater than the differences among the various memory load conditions. In order to find out whether difficulty of the primary task significantly affected probe $\mathrm{RT}$, a second ANOVA was done, omitting the control condition. This analysis provides a stronger test of the assertion that primary and secondary tasks compete for resources (Roediger, Knight, \& Kantowitz, 1977). Again, both main effects and the interaction were significant [for memory load, $\mathrm{F}(4,140)=10.5, \mathrm{MSe}=$ $3,826, \mathrm{p}<.001$; for probe task, $\mathrm{F}(1,35)=21$, MSe $=$ $26,538, p<.001 ;$ for the interaction, $\mathrm{F}(4,140)=2.7$, $\mathrm{MSe}=3,067, \mathrm{p}<.05]$.
RTs from the visual-manual and auditory-vocal probe tasks were also analyzed separately. For these analyses, RT in the secondary task control conditions was again omitted. In the case of the visual-manual probe task, the effect of memory load was significant $[\mathrm{F}(4,140)=9.9$, $\mathrm{MSe}=3,728, \mathrm{p}<.001]$. Paired comparisons among the means showed that RT in the two- and three-item memory load conditions was significantly faster than RT in the five- and seven-item conditions ( $<<.001$, Duncan's multiple-range test). In the case of the auditory-vocal probe task, the effect of memory load was also significant $[\mathrm{F}(4,140)=3.7, \quad \mathrm{MSe}=3,165$, $\mathrm{p}<.01]$. In this case, however, only the difference between RT in the two-item condition and RT in the four- and five-item conditions was significant $(\mathrm{p}<.05)$.

Recall. For each subject, proportion of items correctly recalled was computed for each condition. Mean recall scores across subjects are shown in Figure 2. Recall data were analyzed using a repeated-measures ANOVA in which the within-subjects factors were memory load (two, three, four, five, or seven items) and probe task (no-probe control, visual-manual probe task, or auditoryvocal probe task). Both main effects and the interaction were significant [for memory load, $F(4,140)=202$, MSe $=.016, p<.001$; for probe task, $F(2,70)=19$, $\mathrm{MSe}=.021, \mathrm{p}<.001 ;$ for the interaction, $\mathrm{F}(8,280)=$ $4.8, \mathrm{MSe}=.008, \mathrm{p}<.001]$. In order to explore further the source of the interaction between memory load and probe task, two further ANOVAs were carried out. First, performance in the two probe conditions was combined and compared with performance in the control condition. Again, both main effects and the interaction were significant [for memory load, $F(4,140)=182, \mathrm{MSe}=$ $.011, \mathrm{p}<.001$; for probe task, $\mathrm{F}(1,35)=40$, MSe $=$ $.014, p<.001$; and for the interaction, $F(4,140)=5.5$, $\mathrm{MSe}=.006, \mathrm{p}<.001]$. The effect of the probe task on

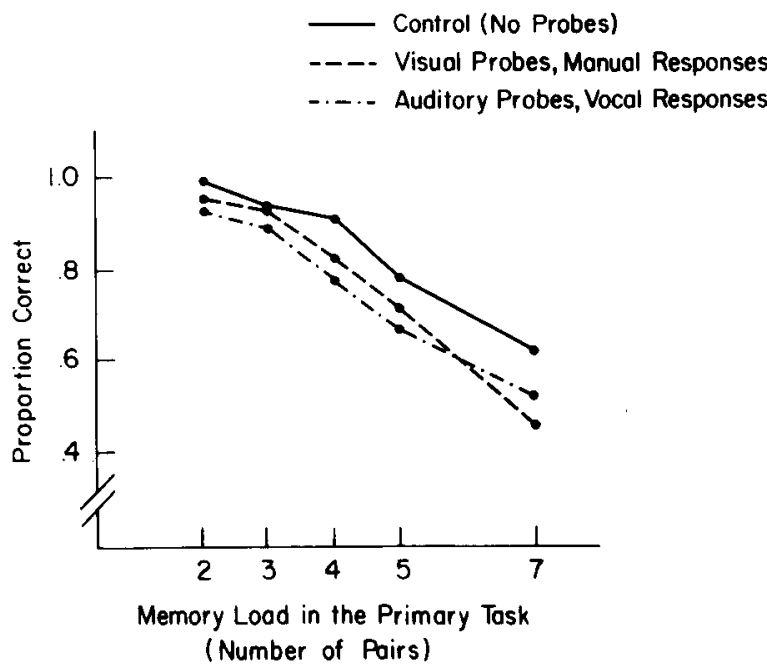

Figure 2. Proportion of items correctly recalled in the control and dual task conditions as a function of memory load, Experiment 1 . 
recall increased as memory load increased. In a second analysis, the two probe conditions were compared, omitting the control condition. Here, the effect of memory load and the interaction between memory load and probe task were significant [for memory load, $F(4,140)=179, \mathrm{MSe}=.014, \mathrm{p}<.001$; for the interaction, $\mathrm{F}(4,140)=4.1, \mathrm{MSe}=.009, \mathrm{p}<.005]$, but the main effect of probe type was not $[F(1,35)=2.0$, $\mathrm{MSe}=.024, \mathrm{p}>.10]$. This interaction evidently reflects the fact that recall was better during the visual-manual probe tasks for all but the seven-item memory load, for which recall was better during the auditory-vocal probe task.

If probe RT is to be used as a measure of spare capacity associated with the paired associate primary task, then there must be evidence that the two tasks compete for mental resources. Experiment 1 provided such evidence. For both probe tasks, RT increased markedly from the control condition to the dual task conditions. Furthermore, RT increased as the demands of the paired associate task were increased. Thus, the experiment supported the assertion that the primary paired associate task and the two secondary probe tasks compete for mental resources.

Ideally, if a secondary task is to serve as a measure of spare capacity, then performance on the primary task should be unaffected by the introduction of the secondary task (Kerr, 1973). In practice, however, this condition is rarely met. Although subjects are instructed not to allow the secondary task to interfere with performance of the primary task, it is common to find a decrement in primary task performance in the dual task conditions as compared with a control condition. Such a decrement occurred in this experiment. Summed over all memory load conditions, proportion of items correctly recalled dropped from .84 in the control condition to .78 in the visual probe condition and .75 in the auditory probe condition. Although significant, the drop was not large and does not negate the validity of the probe task as a measure of spare capacity.

An interesting result of this experiment was that the visual-manual probe task was more sensitive to the demands of the primary task than was the auditory-vocal probe task. This ran counter to our intuitions that the auditory-vocal probe task and verbal rehearsal would compete for a common system. The finding can be explained within a general resource model of attention by supposing that the primary task and both secondary tasks draw from the same common resource pool but that the two secondary tasks have different performanceresource functions (Norman \& Bobrow, 1985). Performance on the visual-manual probe task declines more rapidly than performance on the auditory-vocal task as resources are removed.

Finally, it should be noted that for both secondary tasks, probe RT increased much more from the control condition to the two-item memory load than from the two-item load to the seven-item load. It is a common finding in the dual task literature that there is a large decrement in secondary task performance from control to dual task conditions, but little or no change with increasing difficulty of the primary task (Wickens, 1980). One explanation, proposed by Kantowitz and Knight (1976) and Navon and Gopher (1979), is that coordinating the two tasks demands resources beyond the requirements of each individual task. Thus, the decrement in secondary task performance between control and dual task conditions reflects not only the demands of the primary task, but also the additional demands of coordination.

\section{EXPERIMENTS 2 AND 3}

Experiment 1 demonstrated that probe RT does reflect the difficulty, and thus presumably the resource demands, of the continuous paired associate primary task. It was therefore reasonable to suppose that probe RT would also reflect differences between individuals in the spare capacity associated with the paired associate task. Experiments 2 and 3 were designed to test the validity of the easy-to-hard prediction, that is, to discover whether probe RT during an easy (two-item load) version of the paired associate task would predict performance on a much harder (seven-item load) version of the same task.

Experiment 2 involved the visual-manual probe task, and Experiment 3 involved the auditory-vocal task. In each case, subjects performed the probe task in a control condition, in which they were not asked to recall the letter-digit pairs, and during the rehearsal periods of both the easy and the hard versions of the paired associate task. RT to the probe during the easy paired associate task was taken to be a measure of the spare capacity available to the individual during this task. This measure was then correlated with performance on the harder version of the paired associate task when performed alone. In order to control for individual differences in speed of responding to the probe alone, RT in the control condition was partialled out.

The design of experiments investigating differences between subjects is necessarily quite different from the design of experiments investigating general effects across subjects. It is necessary that enough observations be obtained that the mean observation for each individual in each condition is a reliable estimate of that subject's ability. For this reason, the number of conditions was reduced from 17 in Experiment 1 to only 5 each in Experiments 2 and 3 . To assure that measures were comparable across subjects, the order of conditions was the same for each subject. Thus, these experiments did not utilize a completely counterbalanced design. Finally, in an effort to control motivation, rewards were offered for good performance.

\section{Method}

Subjects. Twenty-four male and 24 female freshmen at the University of Washington served as subjects in Experiment 2. In Experiment 3 , there were 24 males and 26 females. In each case, subjects were selected on the basis of verbal ability. Washington 
state high school students who plan to apply for admission to the University of Washington take the Washington Pre-College Test in their junior year. The distribution of verbal composite scores in the freshman class at the University of Washington was divided into sixths. Approximately four men and four women from each sixth were recruited as subjects in these experiments.

Subjects were paid $\mathbf{\$ 8}$ for participation in two sessions. Bonus points were awarded on the basis of performance in the experimental tasks, and each subject received a bonus payment based on points earned.

Primary and secondary tasks. The tasks were the same as those in Experiment 1, with the following exceptions: (1) Each experiment involved only one type of probe task-for Experiment 2, manual response to a visual probe, and for Experiment 3, vocal response to an auditory probe; (2) there were only two levels of the primary task-subjects were required to keep track of two or seven letter-digit pairs.

Procedure. Subjects were tested on 2 days. On both days, there was one block of 48 trials for each of the five conditions listed below.

Secondary control. Subjects were instructed to ignore the letters and numbers and respond only to the probe stimuli. Letter-number pairs and questions appeared exactly as in other conditions, but questions remained on the screen for only $1 \mathrm{sec}$ Bonus points were based on mean RT to probes.

Easy recall/no probes. Subjects were required to keep track of two pairs. No secondary probes occurred. Points in this condition were based on percentage of letter-digit pairs correctly recalled.

Easy recall with probes. The easy version of the paired associate task was combined with the secondary RT task. Subjects were instructed that the recall task was more important than the RT task. Points in this condition were based on percent recall and mean RT to probes, with twice as many points possible for recall.

Hard recall/no probes. Subjects were required to keep track of seven pairs. No secondary probes appeared. Points were based on percentage of items correctly recalled.

Hard recall with probes. The hard version of the paired associate task was combined with the secondary RT task. Points were based on percentage of items correctly recalled and mean RT to probes, with twice as many points possible for recall.

The order of conditions was identical for all subjects and is shown in Table 2 .

\section{Results and Discussion}

The results of Experiments 2 and 3 will be presented separately. For each experiment, group results summed over individual subjects will be presented first, followed by correlational analyses of individual differences.

Experiment 2. Group results. Seven measures were computed for each subject: mean RT in the control, easy recall, and hard recall conditions, and proportion of items correctly recalled in the easy recall alone, easy recall with probes, hard recall alone, and hard recall with probes conditions. These measures were summed across the 2 days of the experiment. Mean RT summed over all 48 subjects is shown in Figure 3. Mean recall accuracy is shown in Figure 4.

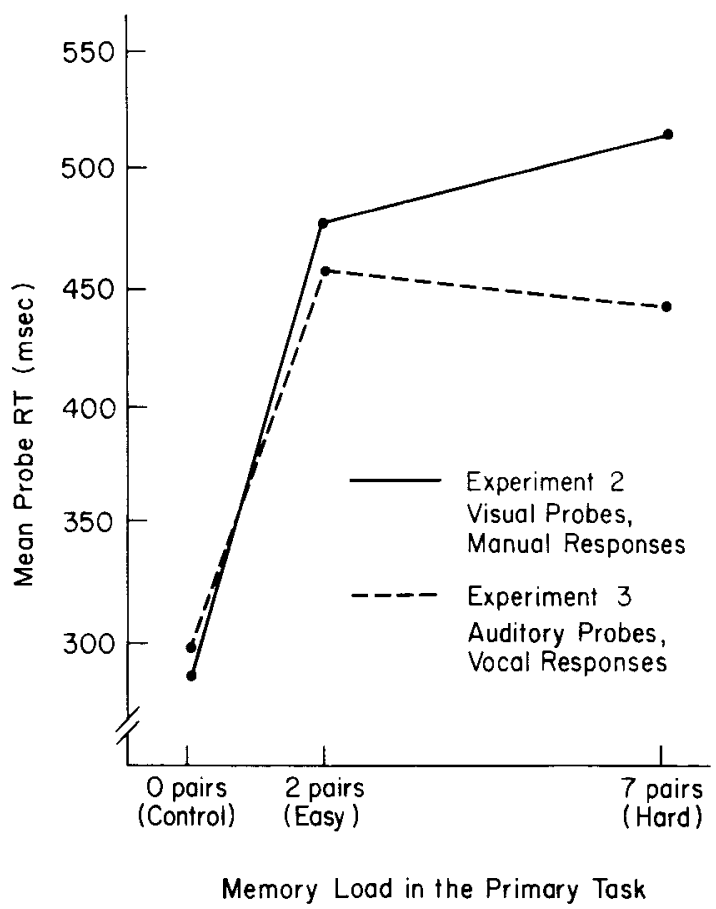

Figure 3. Mean probe RT as a function of memory load in the primary task in Experiment 2 (visual-manual probe task) and Experiment 3 (auditory-vocal probe task).

As in Experiment 1, there was a much greater increase in probe RT from the control to the easy recall condition $(192 \mathrm{msec})$ than from the easy to the hard recall condition $(35 \mathrm{msec})$. An ANOVA showed the effect of recall condition on probe $\mathrm{RT}$ to be significant $[F(2,94)=125, \mathrm{MSe}=5,717, \mathrm{p}<.001]$. Planned orthogonal comparisons revealed that probe RT was shorter in the control condition than in the easy and hard recall conditions combined $[\mathrm{t}(94)=15.7$, $\mathrm{p}<.001$ ] , and probe RT was shorter in the easy recall condition than in the hard recall condition $[\mathrm{t}(94)=$ $2.27, p<.05]$. However, since the order of conditions was the same for all subjects on both days (control-easyhard), these effects were confounded with practice.

Recall was much less accurate in the seven-item condition than in the two-item condition. Proportion of items correctly recalled was above .9 in the twoitem conditions and near .5 in the seven-item conditions $[\mathrm{F}(1,47)=709, \mathrm{MSe}=.011, \mathrm{p}<.001]$. As in Experiment 1, the probe task interfered somewhat with recall, as indicated by the fact that proportion of items correctly recalled was lower in the probe than in the no-probe conditions $[\mathrm{F}(1,47)=32, \mathrm{MSe}=.003$,

Table 2

Order of Conditions in Experiments 2 and 3

\begin{tabular}{llllll}
\hline & Block 1 & \multicolumn{1}{c}{ Block 2 } & Block 3 & Block 4 & Block 5 \\
\hline Day 1 & RT Control & Easy Recall/No Probes & Easy Recall With Probes & Hard Recall/No Probes & Hard Recall With Probes \\
Day 2 & RT Control & Easy Recall With Probes & Easy Recall/No Probes & Hard Recall With Probes & Hard Recall/No Probes \\
\hline
\end{tabular}




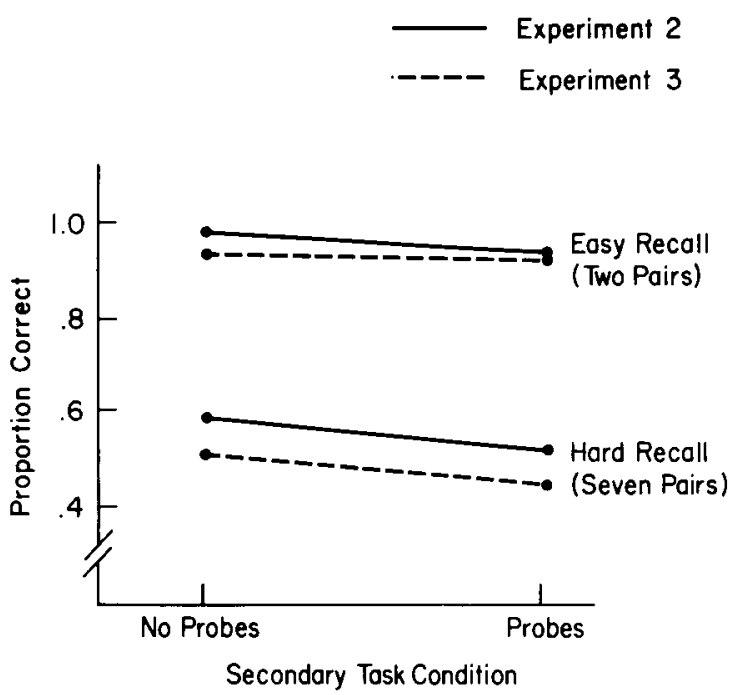

Figure 4. Proportion of items correctly recalled in the easy and hard versions of the primary task as a function of probe condition, Experiments 2 and 3.

$p<.001]$. The interaction between difficulty of the paired associate task and the probe/no-probe factor was also significant $[\mathrm{F}(1,47)=7.0, \mathrm{MSe}=.002, \mathrm{p}<.05]$, indicating that the probe task interfered slightly more with the hard than with the easy version of the paired associate task.

Individual differences. Correlations among the experimental measures are presented in Table 3 , with the reliability of each measure shown in the diagonal. Reliabilities were based on correlations between measures from Day 1 and Day 2, corrected for length using the Spearman-Brown formula.

First, consider the correlations between RT in the control condition and RT in the two dual task conditions (.52 and .36). These correlations are considerably lower than the reliabilities of the measures and indicate that the factors that determined RT in the dual task conditions were not identical to the factors that determined RT in the control condition. This finding is consistent with the hypothesis that the capacity demands of the primary task at least partially determine RT in the dual task conditions.
Next, consider the correlations between RT in the control condition and the four recall measures. These four correlations are near zero, indicating that control $\mathrm{RT}$ is unrelated to ability to recall the paired associate items. These near-zero correlations mean that significant relationships between dual task $\mathrm{RTs}$ and recall measures cannot be attributed to some general ability factor that influences RT and recall in both single task and dual task conditions.

Of greater interest are the correlations between RT during the easy primary task and proportion of items correctly recalled in that task. Assuming that RT is a measure of spare capacity during the easy recall task, one could make two opposing predictions concerning these correlations. One might predict that subjects who expend a large amount of effort on the easy paired associate task would achieve the highest recall scores but would also have the least spare capacity available to respond to the probes. This hypothesis entails a positive correlation between recall and RT: High recall scores should be associated with long RTs. On the other hand, one could argue that subjects who rehearsed the paired associates most efficiently would achieve the highest recall scores and also have the most spare capacity available to respond to the probes. This argument entails a negative correlation between recall and RT in the easy paired associate condition: High recall scores should be associated with short RTs. The correlations in question are -.27 and -.45 . Good performance on the recall task is associated with good performance on the secondary task. These results provide some support for the second argument, that subjects who rehearsed efficiently also had more spare capacity left for the secondary task. They provide no support for the assertion that superior recall is achieved by subjects who exert more effort on rehearsing the letter-number pairs. Nor is there any support for the contention that superior recall reflects a strategy of allotting a large proportion of total processing resources to the primary task at the expense of the secondary task.

Finally, let us look at the "easy-to-hard" correlation, that is, the correlation between RT during the easy primary task and proportion of items correctly recalled in the hard primary task without probes. This correlation is -.40 . The RT measure of spare capacity during

Table 3

Correlation Matrix, Experiment 2

\begin{tabular}{|c|c|c|c|c|c|c|c|}
\hline & 1 & 2 & 3 & 4 & 5 & 6 & 7 \\
\hline $\begin{array}{l}\text { 1. RT: Control Condition } \\
\text { 2. RT: Easy Recall Condition } \\
\text { 3. RT: Hard Recall Condition } \\
\text { 4. PC: Easy Recall/No Probes } \\
\text { 5. PC: Easy Recall With Probes } \\
\text { 6. PC: Hard Recall/No Probes } \\
\text { 7. PC: Hard Recall With Probes }\end{array}$ & $\begin{array}{r}.76 \\
.52 \\
.36 \\
-.09 \\
-.04 \\
-.05 \\
-.03\end{array}$ & $\begin{array}{r}.90 \\
.75 \\
-.27 \\
-.44 \\
-.40 \\
-.37\end{array}$ & $\begin{array}{r}.94 \\
.01 \\
-.14 \\
.07 \\
.06\end{array}$ & $\begin{array}{l}.63 \\
.67 \\
.52 \\
.44\end{array}$ & $\begin{array}{l}.80 \\
.59 \\
.61\end{array}$ & $\begin{array}{l}.74 \\
.85\end{array}$ & .83 \\
\hline
\end{tabular}

Note $-R T=$ probe reaction time; $P C=$ proportion correct. With 48 subjects, correlations of .28 and .36 are significant at the .05 and .01 levels (two-tailed test). 
the easy version of the primary task predicts performance on the harder version. Considering the matter a little more closely, we can think of RT to the probe during the easy memory task as being made up of two components: time to respond to the probe alone, and a delay attributable to the demands of the paired associate task. Only the delay component should reflect the spare capacity associated with the recall task. If the delay is long, then presumably the subject had little spare capacity available during the paired associate task; if it is short, the subject had more spare capacity available. Ideally, we would like to remove from the RT measure the variability associated with individual differences in responding to the probe alone and look only at the delay attributable to the paired associate task. One way to do this is to compute the correlation between probe RT and hard recall, partialling out the variance associated with control RT. This partial correlation was -.44 for hard recall without probes. Thus, even when the variability associated with control RT was removed, RT in the easy recall condition was significantly correlated with the accuracy of recall in the hard condition.

As a practical matter, one might ask whether the probe measure actually improved prediction of performance on the hard recall task above that provided by performance on the easy recall task. In designing this experiment, we expected the easy version of the paired associate task to be so simple that all subjects would perform virtually without error. In that case, performance on the easy version would be a poor predictor of performance on the hard version. In fact, although the mean score on the easy version of the task without probes was .96 , there was some variability, and the correlation between the easy and hard versions without probes was .52. However, when performance in the easy version was partialled out, the easy-to-hard correlation was still significant $(-.32 ; \mathrm{p}<.05)$. When both control RT and performance on the easy version of the primary task alone were partialled out, the easy-to-hard correlation was $-.36(\mathrm{p}<.01)$. Thus, the secondary task provided information not available from either single task measure alone or the two taken together.

The correlations between probe RT during the hard primary task and all four recall measures were near zero. Why were these correlations consistently lower than those involving probe RT during the easy primary? We have argued that probe RT reflects the availability of mental capacity during the primary task. Our conjecture is that, as a measure of spare capacity, probe RT reached a ceiling during the hard paired associate task. Proportion correct on the hard task averaged .54 , and subjects found it extremely demanding. In Experiment 1, RT did not increase between the five-item and seven-item loads. If probe RT did indeed reach a ceiling in this condition, and thus did not reflect individual differences in spare capacity, then it could not be expected to be correlated with the recall measures.
Experiment 3. Group results. The design of Experiment 3 was identical to that of Experiment 2, except that the probe task involved a vocal response to an auditory probe. As in Experiment 2, three RT and four recall measures were computed for each subject. Mean RT measures are shown in Figure 3 and mean recall measures in Figure 4.

As in Experiment 2, RT increased markedly (159 msec) from the control to the easy recall condition. However, there was no increase (actually a decrease of $16 \mathrm{msec}$ ) from easy to hard recall conditions. An ANOVA on probe RT showed the effect of recall condition to be significant $[F(2,98)=193, \quad M S e=1,989, p<.001]$. Planned orthogonal comparisons showed that probe RT was shorter in the control condition than in the easy and hard conditions combined $[\mathrm{t}(98)=19.6, \mathrm{p}<.001]$, but probe $\mathrm{RT}$ in the easy recall condition was not significantly different from probe RT in the hard recall condition $[\mathrm{t}(98)=1.79, \mathrm{p}<.10]$.

Since the effects of the primary task on probe RT were confounded with the effects of practice, it is possible that the effects of practice canceled out the effects of primary task difficulty on probe RT. In any case, it is clear that the effect of the difficulty of the paired associate task on probe RT was stronger in Experiment 2 than in Experiment 3. In this respect, the results of Experiments 2 and 3 are consistent with the results of Experiment 1. Both show that the visualmanual probe task was more sensitive to the demands of paired associate rehearsal than was the auditory-vocal probe task.

As in Experiments 1 and 2, the probe task interfered somewhat with recall. Analysis of recall scores showed both the effect of difficulty and the effect of the probe/ no-probe manipulation to be significant $[F(1,49)=$ $1,686, \mathrm{MSe}=.006, \mathrm{p}<.001$, and $\mathrm{F}(1,49)=44, \mathrm{MSe}=$ $.002, \mathrm{p}<.001]$. The interaction was also significant $[\mathrm{F}(1,49)=12.8, \mathrm{MSe}=.002, \mathrm{p}<.001]$, indicating that the probe task interfered more with hard than with easy recall.

Individual differences. Correlations among experimental measures are shown in Table 4, with reliabilities in the diagonal. As in Experiment 2, correlations between control RT and RT in the dual task conditions were considerably lower than the reliabilities of the measures. The capacity demands of the primary task seem to have influenced individual performance on the RT task in the dual conditions.

However, in Experiment 3, unlike in Experiment 2, there was a positive relationship between control RT and paired associate recall. The correlation between control RT and recall in the hard paired associate task without probes was significant $(\mathrm{r}=.37, \mathrm{p}<.01)$. Vocal RT to an auditory probe was positively related to the ability to recall the paired associates. This was not the case for manual RT to a visual probe in Experiment $2 .^{2}$ The relationship between control RT and recall in 
Table 4

Correlation Matrix, Experiment 3

\begin{tabular}{llllllll}
\hline & 1 & 2 & 3 & 4 & 5 & 6 & 7 \\
\hline 1. RT: Control Condition & .84 & & & & & & \\
2. RT: Easy Recall Condition & .40 & .84 & & & & & \\
3. RT: Hard Recall Condition & .37 & .60 & .77 & & & & \\
4. PC: Easy Recall/No Probes & -.13 & -.12 & -.08 & .60 & & & \\
5. PC: Easy Recall With Probes & -.15 & -.19 & -.07 & .80 & .72 & .63 & \\
6. PC: Hard Recall/No Probes & -.37 & -.39 & -.16 & .28 & .25 & .63 & .46 \\
7. PC: Hard Recall With Probes & -.22 & -.20 & .18 & .40 & .32 & .59 &
\end{tabular}

Note-RT = probe reaction time; $P C=$ proportion correct. With 50 subjects, correlations of .27 and .35 are significant at the .05 and .01 levels (two-tailed test).

Experiment 3 clouds interpretation of correlations involving probe $\mathrm{RT}$ in the dual task conditions.

The easy-to-hard correlation was -.39 in Experiment 3. This correlation was almost identical to the correlation based on RT in the control condition (-.36). Can this correlation be taken as support for the easy-tohard prediction? RT increased from $300 \mathrm{msec}$ in the control condition to $459 \mathrm{msec}$ in the dual task condition. The question is whether the variability in this increase was associated with performance on the hard recall task. The question can be answered by examining the partial correlation between RT during easy recall and accuracy on the hard recall task, holding control RT constant. This partial correlation was $-.29(\mathrm{p}<.01)$, providing support for the easy-to-hard prediction. Thus, the results of Experiment 3 can be interpreted as support for the hypothesis that spare capacity during an easy primary task predicts performance on a harder version of the same primary task. However, the support is less clear-cut than in Experiment 2.

Finally, does the probe RT measure improve prediction of performance on the hard recall task beyond that provided by performance on the easy recall task? The correlation between RT during the easy task and performance on the hard task, partialling out performance on the easy primary alone, was significant $(r=-.37$, $\mathrm{p}<.01)$. It remained significant even when control RT was partialled out as well $(\mathrm{r}=-.28, \mathrm{p}<.05)$. The dual task measure predicted performance on the hard primary task even when the effects of both single task measures were removed.

\section{EXPERIMENT 4}

Experiment 4 involved both the paired associate and the spatial memory primary tasks. The main purpose was to find out whether the correlational results of Experiments 2 and 3 could be extended to a spatial memory task. A second purpose was to find out whether retention of spatial information would interfere as much with response to the probe as paired associate rehearsal did. Although both tasks involve short-term memory, they seem to require entirely different memorization strategies and, conceivably, different amounts of mental effort.

\section{Method}

Subjects. Eighty-one subjects, 52 female and 29 male, ranging in age from 18 to 60 years participated in this study. They were recruited through an ad in the university newspaper and were paid $\$ 4.50$ per $1.5-\mathrm{h}$ session for five sessions. The participation of some older nonstudents as subjects considerably broadened the range of performance in this experiment as compared with the previous three. The first three sessions of the study involved a dichotic listening paradigm that will not be reported here.

Tasks. Spatial memory: Primary task. The spatial memory task is illustrated in Figure 5. At the beginning of each trial, a single plus sign was shown in the center of the screen for $1 \mathrm{sec}$. Then a standard pattern appeared on the screen. In the easy version of the task, the standard pattern was formed by placing pluses in four positions randomly selected from a 3 by 3 matrix. In the hard version of the task, the standard pattern was formed by placing 10 pluses in a 7 by 7 matrix. The standard pattern remained on the screen for $3 \mathrm{sec}$, during which time the subject was instructed to study and memorize it. After $3 \mathrm{sec}$, the pattern was replaced by the entire matrix of pluses. This mask remained on the screen for $1 \mathrm{sec}$. Finally, a test pattern was shown. The test pattern was either identical to the standard pattern or

Event
Standard pattern
500,1000, or 1500
msec after onset of
standard pattern.
Mask.
Test pattern. Subject
responds as to whether
test pottern is the
same or different from
the standard.

Figure 5. Sequence of events in the spatial memory task of Experiment 4. 
slightly different. ("Different" patterns were formed by moving one plus one space up, down, to the right, or to the left.) The subject responded "same" if the test pattern was identical to the standard and "different" if it was not. "Same" responses were made with the right index finger and "different" responses with the right middle finger. There was no time pressure to respond to the test pattern.

Spatial memory: Secondary task. The secondary task in the spatial memory paradigm involved responding to an auditory probe stimulus that occurred during the $3-\mathrm{sec}$ study phase of the primary task. The probe was a $100-\mathrm{msec}$ tone, and the response was a keypress. Whereas probes in the previous experiments continued until the subject responded, in this experiment they were terminated automatically after $100 \mathrm{msec}$. Probes occurred equally often $500,1,000$, or $1,500 \mathrm{msec}$ after the onset of the standard pattern. During each 32-trial block, there were eight probes at each interval and 8 catch trials during which no probes occurred. The subject pressed a key with the left index finger as quickly as possible when a probe occurred.

Paired associates: Primary task. The easy version of this task was exactly the same as that used in Experiments 2 and 3; subjects kept track of two letter-digit pairs. In the hard version, subjects kept track of five pairs.

Paired associates: Secondary task. The secondary probe task was identical to that used in Experiments 2 and 3, except that the probe was a $100-\mathrm{msec}$ tone and the response was a keypress. Probes occurred equally often $500,1,000$, or $1,500 \mathrm{msec}$ after the onset of a new letter-digit pair. In each 32-trial block, eight probes occurred at each interval, and there were 8 catch trials on which no probe occurred.

Procedure. Day 4 of the study was devoted to the spatial memory task, and Day 5 to the paired associate task. There were five conditions for each of the two tasks.

Secondary control. Subjects responded only to the probe tones. Stimuli for the primary task were presented, but subjects did not respond to them.

Easy primary/no probes. The easy primary task was presented alone. No probes occurred.

Easy primary with probes. The easy version of the primary task was combined with the secondary probe RT task.

Hard primary/no probes. The hard primary task was presented alone. No probes occurred.

Hard primary with probes. The hard version of the primary task was combined with the secondary probe RT task.

On each of the 2 days, there were 10 blocks of 32 trials each, 2 blocks for each of the five conditions listed above. In the first five blocks, the conditions were presented in the order listed above, and in the last five blocks, this order was reversed.

\section{Results and Discussion}

Group results. Seven measures were computed for each subject on each task: mean probe RT in the control, easy primary, and hard primary conditions and proportion of correct responses to the primary task in the easy condition alone, easy condition with probes, hard condition alone, and hard condition with probes. These measures were summed across the two blocks of each condition. Mean probe RT is shown in Figure 6, and mean proportion of correct responses is shown in Figure 7.

Probe RT was analyzed using a repeated-measures ANOVA in which the factors were primary task type (paired associate or spatial memory) and primary task difficulty (control, easy primary, or hard primary). The main effect of primary task type was significant $[\mathrm{F}(1,80)=227, \mathrm{MSe}=14,702, \mathrm{p}<.001]$, indicating that

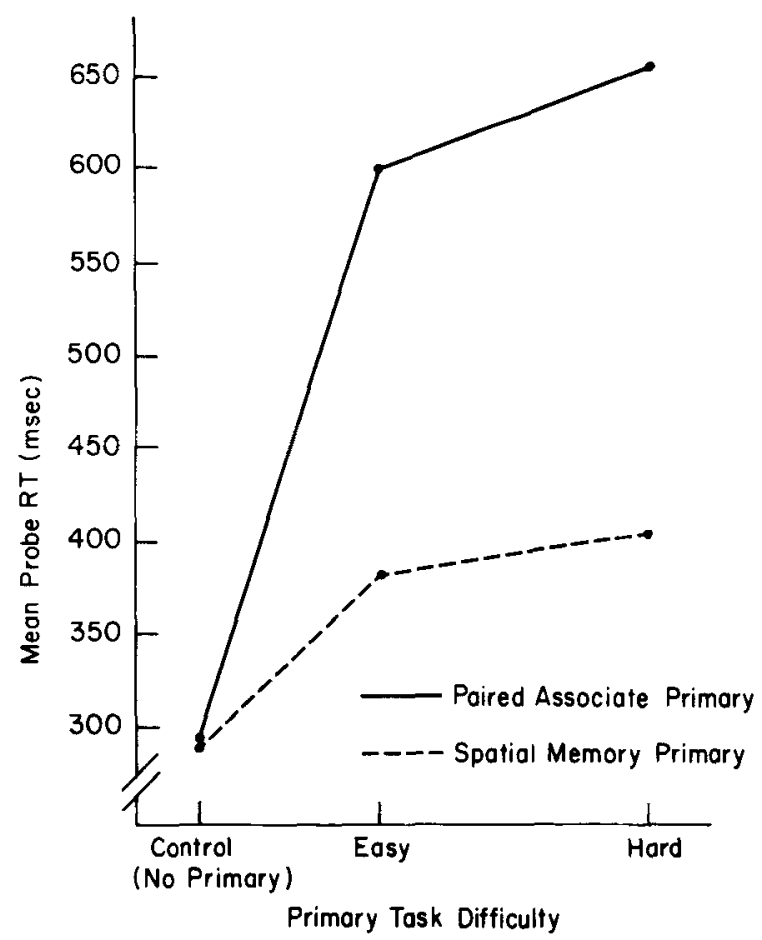

Figure 6. Mean probe RT during paired associate and spatial memory primary tasks as a function of primary task difficulty, Experiment 4.

RT to the probe was longer during the paired associate than during the spatial memory primary task. The main effect of primary task difficulty was also significant $[\mathrm{F}(2,160)=279, \mathrm{MSe}=10,119, \mathrm{p}<.001]$, as was the interaction $[F(2,160)=121, \mathrm{MSe}=6,414, \mathrm{p}<.001]$. RT increased with the demands of the primary tasks, and this effect was stronger during the paired associate task than during the spatial memory task.

Probe RTs were also analyzed separately for each of the primary tasks. For the spatial memory task, the effect of primary task difficulty was again significant $[F(2,160)=92, \mathrm{MSe}=3,554, p<.001]$. Planned orthogonal comparisons on spatial memory RTs showed that RT was shorter in the control condition than in the easy and hard conditions combined $[t(160)=13.0$, $\mathrm{p}<.001]$ and $\mathrm{RT}$ was shorter in the easy recall condition than in the hard recall condition $[\mathrm{t}(160)=4.12$, $p<.001]$. For the paired associate task, the effect of primary task difficulty was also significant $[F(2,160)=$ $252, \mathrm{MSe}=12,979, \mathrm{p}<.001 \mathrm{]}$. Again, planned orthogonal comparisons showed that RT was shorter in the control condition than in the easy and hard conditions combined $[\mathrm{t}(160)=22.2, \mathrm{p}<.001]$ and $\mathrm{RT}$ was shorter in the easy recall condition than in the hard recall condition $[\mathrm{t}(160)=3.15, \mathrm{p}<.01]$.

In Experiments 1, 2, and 3, in which probes were terminated by the subject's response, less than $1 \%$ of the probes were ignored. In Experiment 4, in which the duration of the probe was $100 \mathrm{msec}$ regardless of the 


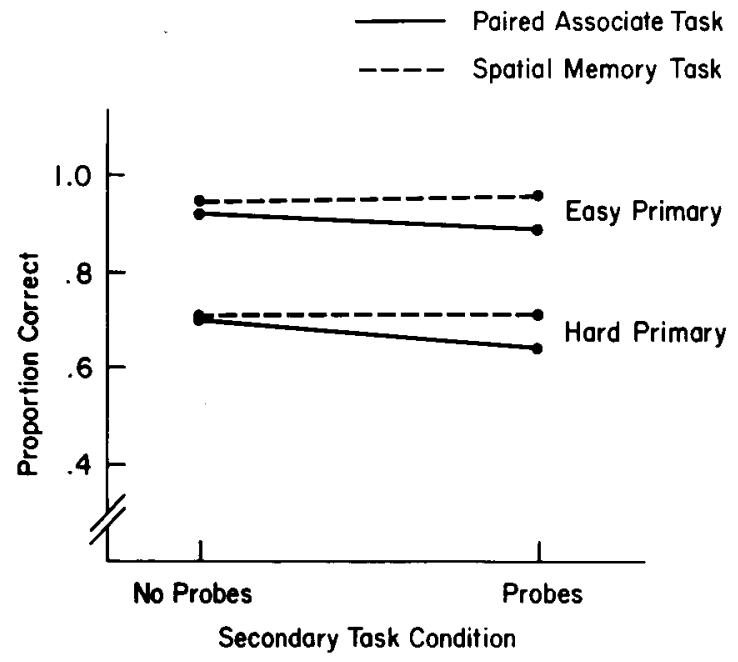

Figure 7. Proportion of correct responses to the two primary tasks as a function of probe condition, Experiment 4.

subject's response, many more probes were ignored. For the spatial memory primary task, $2 \%$ of the probes were ignored in the control condition, $2 \%$ during the easy primary, and 3\% during the hard primary. For the paired associate primary task, $1 \%$ of the probes were ignored in the control condition, $8 \%$ during the easy primary, and $12 \%$ during the hard primary. Thus, the data on ignored probes are consistent with the RT data in showing that the paired associate task interfered more with response to the probes than did the spatial memory task.

Accuracy of responses to the primary task also indicated that there was more interference between the probe task and the paired associate task than between the probe task and the spatial memory task. Accuracy scores from the two primary tasks were analyzed separately, since they were not directly comparable. (Chance performance in the spatial memory task was .50, whereas chance performance in the paired associate task was .125.) In each case, a repeated-measures ANOVA was performed in which the two factors were difficulty of the primary task (easy or hard) and probe condition (probes or no probes). For the spatial memory task, only the main effect of difficulty was significant $[F(1,80)=$ $1,513, \mathrm{MSe}=.003, \mathrm{p}<.001]$. Neither the main effect of probe condition nor the interaction was significant. Probes did not interfere with accuracy of responses to the spatial memory task. In the analysis of paired associate recall, both the main effect of difficulty $[F(1,80)=$ $214, \mathrm{MSe}=.020, \mathrm{p}<.001]$ and the main effect of probe condition $[\mathrm{F}(1,80)=48, \mathrm{MSe}=.005, \mathrm{p}<.001]$ were significant. The interaction was only marginally significant $[\mathrm{F}(1,80)=3.6, \mathrm{MSe}=.005, \mathrm{p}<.10]$. In the case of the paired associate task, the probes did interfere with the accuracy of recall, and this effect was slightly greater for the hard than for the easy version.
The paired associate data from Experiment 4 replicate those of the previous experiments. Paired associate rehearsal caused a significant delay in responding to the probes, and the probes also caused some decrement in accuracy of recall. Memorization of the spatial patterns caused much less of a delay in responding to the probes, and accuracy of response to the spatial patterns was unaffected by the probes. The most obvious interpretation of the fact that there was less interference between the spatial memory and the probe tasks is that subjects devoted less effort to studying the spatial patterns than to rehearsing the paired associates. Why should this be the case? Perhaps it was because an active verbal strategy can be employed to maintain the paired associates, but no similar strategy is available for the spatial memory task. Subjects can improve their performance on the paired associate task by devoting more mental resources to rehearsal. There is no similar method of utilizing mental resources to improve performance on the spatial memory task. This explanation is consistent with introspection concerning the two tasks. Subjects spoke of being exhausted by the hard paired associate task. But they reported that a passive attitude was more effective in memorizing the spatial patterns.

Individual differences. Table 5 shows the correlations among measures from both spatial memory and paired associate tasks. Reliabilities are shown in the diagonal. In Experiment 4, reliabilities were computed by correlating odd with even trials and correcting for length using the Spearman-Brown formula.

Consider first the correlations among measures based on the spatial memory task. The correlations between control RT and RT in the two dual task conditions (.76 and .70) are higher than the corresponding correlations for Experiments 2 and 3 and higher than those based on the paired associate task in Experiment 4 (.61 and .49). These higher correlations are consistent with the finding that the spatial memory task had less effect on probe RT than did the paired associate task.

Control RT was also positively and significantly related to all four measures of accuracy on the spatial memory task ( $\mathrm{rs}=-.31,-.49,-.27$, and -.30 ). Furthermore, the correlations between probe RT during the easy primary task and these four accuracy measures were almost identical to the correlations involving control RT ( $\mathrm{rs}=-.31,-.46,-.29$, and -.26 ). The partial correlation between RT during the easy primary and accuracy on the hard primary, removing the effects of control RT, was nonsignificant $(\mathrm{r}=-.14)$. Thus, the easy-to-hard prediction was not supported when the effects of RT in the control condition were removed. ${ }^{3}$

The correlations among measures based on the paired associate data support a more positive conclusion. As in the spatial memory data, control RT was positively related to the four measures of accuracy on the primary task. However, correlations between RT during the easy primary task and these four accuracy measures were con- 
Table 5

Correlation Matrix, Experiment 4

\begin{tabular}{|c|c|c|c|c|c|c|c|c|c|c|c|c|c|c|}
\hline & 1 & 2 & 3 & 4 & 5 & 6 & 7 & 8 & 9 & 10 & 11 & 12 & 13 & 14 \\
\hline & \multicolumn{14}{|c|}{ Spatial Memory Task } \\
\hline 1. RT: Control Condition & .99 & & & & & & & & & & & & & \\
\hline 2. RT: Easy Primary Condition & .76 & .99 & & & & & & & & & & & & \\
\hline 3. RT: Hard Primary Condition & .70 & .88 & .97 & & & & & & & & & & & \\
\hline 4. PC: Easy Primary/No Probes & -.31 & -.31 & -.18 & .55 & & & & & & & & & & \\
\hline 5. PC: Easy Primary With Probes & -.49 & -.46 & -.33 & .61 & .61 & & & & & & & & & \\
\hline 6. PC: Hard Primary/No Probes & -.27 & -.29 & -.13 & .27 & .40 & .59 & & & & & & & & \\
\hline \multirow[t]{2}{*}{ 7. PC: Hard Primary With Probes } & -.30 & -.26 & -.18 & .41 & .47 & .48 & .50 & & & & & & & \\
\hline & \multicolumn{14}{|c|}{ Paired Associate Task } \\
\hline 8. RT: Control Condition & .82 & .69 & .63 & -.20 & -.33 & -.34 & -.31 & .99 & & & & & & \\
\hline 9. RT: Easy Recall Condition & .52 & .63 & .55 & -.27 & -.35 & -.27 & -.21 & .61 & .97 & & & & & \\
\hline 10. RT: Hard Recall Condition & .41 & .56 & .52 & -.03 & -.21 & -.27 & -.11 & .49 & .65 & .96 & & & & \\
\hline 11. PC: Easy Recall/No Probes & -.38 & -.53 & -.40 & .57 & .41 & .38 & .29 & -.39 & -.48 & -.11 & .93 & & & \\
\hline 12. PC: Easy Recall With Probes & -.20 & -.39 & -.27 & .46 & .38 & .47 & .50 & -.28 & -.41 & -.04 & .68 & .96 & & \\
\hline 13. PC: Hard Recall/No Probes & -.19 & -.29 & -.20 & .40 & .28 & .27 & .39 & -.21 & -.49 & -.06 & .57 & .73 & .96 & \\
\hline 14. PC: Hard Recall With Probes & -.24 & -.29 & -.18 & .44 & .29 & .30 & .47 & -.26 & -.41 & -.04 & .55 & .69 & .89 & .95 \\
\hline
\end{tabular}

Note-RT = probe reaction time; $P C=$ proportion correct. With 81 subjects, correlations of .22 and .28 are significant at the .05 and .01 levels (two-tailed test).

sistently higher. Specifically, the easy-to-hard correlation was -.49. When control RT was partialled out of this correlation, it remained significant (-.47). As in Experiments 2 and 3 , the easy-to-hard prediction was supported in the paired associate data from Experiment 4.

Why was the easy-to-hard correlation nonsignificant in the case of the spatial memory task? The answer may be related to the resource requirements of that task. We suggested earlier that subjects use a resource-demanding rehearsal strategy to memorize paired associates, but that no such strategy is available for the spatial memory task. For this reason, resource limitations may determine an individual's performance on the paired associate task, but not on the spatial memory task. If availability of resources is not the limiting factor in performing the hard version of the spatial task, then one would not expect a relationship between spare capacity available during the easy version of the task and performance on the harder version.

\section{GENERAL DISCUSSION}

The research reported here can be considered from both a theoretical and a practical point of view. Theoretically, we have argued that a simple model of attention has direct implications concerning individual differences. Practically, the secondary task methodology offers a technique for predicting performance on difficult tasks that cannot be observed directly. The theoretical implications will be considered first, followed by a brief discussion of the practical significance of the research.

The easy-to-hard prediction is based on four assumptions: (1) Primary and secondary tasks compete for a limited attentional resource; (2) performance on both tasks is determined, at least partially, by the availability of this resource; (3) subjects adopt a consistent resource allocation policy in which the primary task is given highest priority; and (4) spare capacity available during the easy primary can be used to meet the increased demands of the hard primary task.

These assumptions lead to the prediction that "spare capacity," as measured by secondary task performance during the easy primary, should predict performance on the hard primary task (i.e., the easy-to-hard prediction). To what extent did the data support this prediction? To answer this question, we first looked at the first-order correlations between probe RT during the easy memory task and accuracy on the hard memory task. These correlations were quite consistent across experiments $(-.40,-.39$, and -.49 on the paired associate task and -.29 on the spatial memory task). However, the firstorder correlations alone are not sufficient to support the argument. Probe RT can be thought of as measuring two individual characteristics: (1) spare capacity associated with the easy memory task and (2) skill specific to the secondary task itself. In order to support our reasoning, we need to show that variability in the first of these characteristics is correlated with the criterion variable.

The case was very clear in Experiment 2, since RT in the control condition was uncorrelated with accuracy in the recall task. In that experiment, the easy-to-hard correlation must have been due to the spare-capacity component of probe RT. In Experiments 3 and 4, the situation was less clear. In each of these cases, control RT was correlated with performance in the hard recall task. Here, we asked whether the easy-to-hard correlation remained even when the variability associated with control RT was partialled out. For the paired associate task, the partial correlation was significant. For the spatial memory task, it was not. 
The paired associate and spatial memory tasks differed along several dimensions: One was verbal, one spatial; one involved recall, the other recognition; one required the subject to maintain a memory load from trial to trial, and in the other, trials were discrete. It is not possible to conclude with certainty why the easy-tohard prediction was significant for one task and not the other. We can, however, review the four assumptions listed above and consider the extent to which these assumptions were met by the paired associate and spatial memory tasks.

The first assumption is that primary and secondary tasks compete for a single resource. There have been several criticisms of this assumption. It could well be that there are several pools of mental resources (Allport, 1980; McLeod, 1977; Navon \& Gopher, 1979; Wickens, 1980). While the easy-to-hard prediction is not based on the assumption that there is only one, general attentional resource, it does require that primary and secondary tasks draw from the same resource pool. This assumption can be tested by examining the pattern of interference between the two tasks. For all primary. secondary pairs in these experiments, secondary RT increased dramatically from single task to dual task conditions and also (with the exception of Experiment 3) increased from the easy to the hard version of the primary task. This result indicates that primary and secondary did compete for resources. However, the interference between tasks was considerably stronger for the paired associate than for the spatial memory task. Thus, it is possible that the resource demands of the probe task overlapped more with those of the paired associate than with those of the spatial memory task.

The second assumption is that performance on both primary and secondary tasks is determined at least partially by the availability of resources, that is, that the tasks are resource limited (Norman \& Bobrow, 1975). A resource-limited task is normally defined as a task on which performance improves with increased allocation of resources. However, in the study of individual differences, "resource limited" may be used in a slightly different sense. Here, to say that a task is resource limited means that the performance of an individual, relative to other individuals, is determined by resource limitations rather than data limitations. Resource availability may be a stronger determinant of performance on the verbal paired associate task, since subjects can utilize available resources for rehearsal. In the spatial task, some other factor, such as durability of a visual image, may be a more important determinant of performance.

The third assumption is that subjects adopt a consistent resource allocation strategy in which the primary task is given top priority. This assumption is perhaps the most questionable of the four. The typical secondary task instructions advise subjects that the primary task is more important than the secondary and warn them not to let the secondary interfere with the primary. The theory assumes that subjects can allocate sufficient resources to the primary so that it can be performed adequately and use what is "left over" on the secondary task. In fact, subjects often find it impossible to prevent the secondary task's interfering with the primary. Under these circumstances, they must choose their own strategy for combining the two tasks, and this strategy may differ across subjects. Some subjects may consider the secondary task "more secondary" than others. To the extent that subjects differ in allocation strategies, the secondary task is an invalid measure of spare capacity.

The final assumption states that spare capacity, as measured by secondary performance during the easy primary, can be used to meet the increased demands of the hard primary. The key point here is that, as the primary task becomes more difficult, resource demands change quantitatively but not qualitatively. Suppose for a moment that there are multiple resource pools. One can imagine a situation in which increasing the difficulty of a task changed the type of resources required by that task. Then spare capacity, as measured by the secondary task, would not be expected to predict performance on the more difficult primary. In these experiments, both paired associate and spatial memory primary tasks were designed to require similar resources at the two difficulty levels.

To summarize, in order for the easy-to-hard prediction to be successful, a number of assumptions must be met. Both tasks must draw upon the same resources. Resource demands must be the same at both levels of difficulty of the primary task. Individual differences in performance on both tasks must be determined to some extent by available resources. And subjects must be fairly consistent in their allocation strategies. The fact that the easy-to-hard prediction was successful for the paired associate task suggests that these assumptions were fulfilled for that task. The failure of the easy-tohard prediction in the case of the spatial memory task can most likely be attributed to the fact that individual performance on that task was not determined by resource availability. Another, quite similar, explanation is that the resource demands of spatial memory and probe tasks did not overlap sufficiently. At this point, either explanation remains quite speculative.

Putting aside theoretical issues, we suggest that the technique presented here has practical implications. There are numerous situations in which one would like to predict a person's response to highly demanding conditions without actually testing under those conditions. For example, a prospective bus driver should be able to handle both routine traffic and the increased demands of an emergency, but job applicants cannot be tested in emergencies. The easy-to-hard technique suggests a method of extrapolating from common to extraordinary conditions.

The idea of using dual task performance as a predictive tool is not new in applied psychology. Applied studies of dual task performance are often predicated on the assumption that there is a general timesharing ability and that this ability, if measurable, should predict per- 
formance on complex tasks, such as airplane piloting. In support of this idea, several studies have shown that piloting ability is better predicted by dual task than by single task measures (Damos, 1978; North \& Gopher, 1976). In fact, however, the evidence for a general timesharing ability is weak. Factor-analytic studies in which dual task and single task measures are analyzed together show little evidence that all dual tasks reflect a single timesharing ability (Jennings \& Chiles, 1977; Sverko, 1977). Ability to do two things at once seems to be specific to the particular task combination tested. (For a review of this issue, see Hawkins, Rodriguez, and Reicher, Note 1.)

Another approach to the prediction of performance in multicomponent jobs is to measure the residual capacity associated with some aspect of the job. Such measures are assumed to estimate the effort that component requires. A number of applied studies have utilized this method (Brown, 1968; Crosby \& Parkinson, 1979; Damos, 1978). For example, Crosby and Parkinson (1979) used a memory-scanning task to measure subjects' residual capacity during a groundcontrolled approach in a flight simulator. They found a huge difference between student pilots and flight instructors in memory-scanning RT in the dual task but not in the single task condition. They argued that for practiced pilots, the ground-controlled approach required less total attentional capacity, leaving more capacity available for the memory task.

The easy-to-hard approach resembles that of Crosby and Parkinson (1979), but there are several differences. Perhaps the most important difference is that we designed our task combinations to reflect central rather than peripheral or "structural" interference. Dual tasks reported in the applied literature often require subjects to shift their eyes from one position to another or to respond nearly simultaneously to two signals. Such dual tasks may measure the subject's ability to develop a specific strategy for combining the primary and secondary tasks, rather than the residual attention associated with the primary task. Second, our criterion measure was performance on a more difficult version of the primary task. The rationale for choosing this criterion follows directly from a resource theory of attention. As far as we know, the easy-to-hard approach has never been tested in the applied area. Third, we attempted a somewhat stronger test of our technique, in that we correlated predictor with criterion variables, rather than attempting to discriminate between extreme ability groups, as many applied studies of dual task performance have done. Our success using a laboratory task suggests that the technique may be applicable in a more applied setting.

\section{REFERENCE NOTE}

1. Hawkins, H. L., Rodriguez, E., \& Reicher, G. M. Is timesharing a general ability? (Tech. Rep. 3). University of Oregon, Center for Cognitive and Perceptual Research, 1979.

\section{REFERENCES}

Allport, D. A. Attention and performance. In G. Claxton (Ed.), Cognitive psychology: New directions. London: Routledge \& Kegan Paul, 1980.

Atkinson, R. C., \& Shiffrin, R. M. Human memory: A proposed system and its control processes. In K. W. Spence \& J. T. Spence (Eds.), The psychology of learning and motivation: Advances in research and theory (Vol. 2). New York: Academic Press, 1968.

Baddeley, A. D. The psychology of memory. New York: Basic Books, 1976.

Baddeley, A. D., Thompson, N., \& Buchanan, M. Word length and structure of short term memory. Journal of Verbal Learning and Verbal Behavior, 1975, 14, 575-589.

Britton, B. K., Westbrook, R. D., \& Holdredge, T. S. Reading and cognitive capacity usage: Effects of text difficulty. Journal of Experimental Psychology: Human Learning and Memory, 1978, 4, 582-591.

Brown, I. D. Some alternative methods of predicting driver performance among professional drivers in training. Ergonomics, $1968,11,13-21$.

Crosby, J. V., \& Parkinson, S. R. A dual task investigation of pilots' skill level. Ergonomics, 1979, 22, 1301-1313.

DAmos, D. L. Residual attention as a predictor of pilot performance. Human Factors, 1978, 20, 435-440.

Jennings, A. E., \& Chiles, W. D. An investigation of timesharing ability as a factor in complex performance. Human Factors, 1977, 19, 535-547.

Johnston, W. A., Greenberg, S. N., Fisher, R. P., \& Martin, D. W. Divided attention: A vehicle for monitoring memory processes. Journal of Experimental Psychology, 1970, 83, 164-171.

Kahneman, D. Attention and effort. Englewood Cliffs, N.J: Prentice-Hall, 1973.

KANTOWITZ, B., \& KNIGHT, J. Testing tapping time-sharing: II Auditory secondary task. Acta Psychologica, 1976, 40, 343362.

KERR, B. Processing demands during mental operations. Memory \& Cognition, 1973, 1, 401-412.

LoGAN, G. D. Attention in character-classification tasks: Evidence for the automaticity of component stages. Journal of Experimental Psychology: General, 1978, 107, 32-63.

Martin, D. W. Residual processing capacity during verbal organization in memory. Journal of Verbal Learning and Verbal Behavior, 1970, 9, 391-397.

McLeod, P. A dual task response modality effect: Support for multiprocessor models of attention. Quarterly Journal of Experimental Psychology, 1977, 29, 651-667.

McLeod, P. What can probe RT tell us about the attentional demands for movement? In G. E. Stelmach \& J. Requin (Eds.), Tutorials in motor behavior. Amsterdam: North Holland, 1980.

Navon, D., \& Gopher, D. On the economy of the humanprocessing system. Psychological Review, 1979, 86, 214-255.

Norman, D. A., \& Bobrow, D. B. On data-limited and resourcelimited processes. Cognitive Psychology, 1975, 7, 44-64.

NoRTH, R. A., \& Gopher, D. Measures of attention as predictors of flight performance. Human Factors, 1976, 18, 1-14.

Posner, M. I., \& Boles, S. J. Components of attention. Psychological Review, 1971, 78, $391-408$.

Roediger, H. L., KNight, J. L., \& Kantowitz, B. H. Inferring decay in short-term memory: The issue of capacity. Memory \& Cognition, 1977, 5, 167-176.

Schwartz, S. P. Capacity limitations in human information processing. Memory a Cognition, 1976, 4, 763-768.

Sverko, B. Individual differences in time-sharing performance. Acta Instituti Psycholigici, 1977, 79, 17-30.

Wickens, C. D. The structure of attentional resources. In R. S. Nickerson (Ed.), Attention and performance VIII. Hillsdale, N.J: Erlbaum, 1980. 


\section{NOTES}

1. It has been argued (a) that mental capacity expands to meet the demands of the task faced by the subject (e.g., Kahneman, 1973) and (b) that subjects differ in the amount of mental capacity they are willing to devote to the experiment during any given condition. There is no feasible way to test these assertions, except perhaps to take physiological measures of arousal, which in themselves are often inconsistent and are beyond the scope of this research. For the purposes of this paper, we will proceed on the alternative assumptions that capacity is fixed and that, for the most part, subjects "try their hardest"' in all experimental conditions.

2. An interesting side question is why vocal RT to an auditory stimulus should be correlated with recall, when manual RT to a visual stimulus was not. Baddeley's assertion that an individual's immediate memory span is determined by the speed with which the person can pronounce the items to be recalled provides a possible link (Baddeley, Thompson, \& Buchanan, 1975). It is tempting to speculate that the speed of vocal response is related to the speed of rehearsing a series of words or numbers, which, in turn, determines recall accuracy in the paired associate task.

3. A problem arises in interpreting correlations involving proportion of correct responses in the hard spatial memory task, since the reliability of that measure was only .59. In order to increase the reliability, proportion correct in the probe and noprobe conditions were combined. This seemed justified, since probes had no effect on mean accuracy scores for the spatial task, and because the patterns of correlations involving accuracy in probe and no-probe conditions were very similar. The reliability of the new measure was .73. The correlation between the combined accuracy measure and RT during the easy spatial memory task was $-.32(p<.01)$, but the correlation with control RT was $-.33(\mathrm{p}<.01)$. The partial correlation between the combined accuracy measures and RT during the easy version, removing the effects of control RT, was $-.12(p>.10)$. Thus, the failure of the easy-to-hard correlation in the case of the spatial memory task can probably not be attributed to the unreliability of the accuracy measure.

(Received for publication April 29, 1981; revision accepted October 20,1981.) 